\title{
Bilateral Cervical Lymphangioma of The Neck In An Adult: A Rare Case with Review of Literature
}

\author{
Jayesh Sardhara ${ }^{1}$, Anant Mehrotra ${ }^{1}$, Amit Kumar Singh ${ }^{1}$, Pavaman Sindgikar ${ }^{1}$, \\ Mukesh Shukla ${ }^{1}$, Puja Chovatiya ${ }^{2}$, Kamlesh Bhaishora ${ }^{1}$, Kuntal K Das ${ }^{1}$, Arun \\ Srivastava ${ }^{1}$, Sanjay Behari ${ }^{1}$ \\ ${ }^{1}$ (Department Of Neurosurgery, Sanjay Gandhi Post Graduate Of Medical Sciences, Lucknow, UP, India) \\ ${ }^{2}(2$ Dr. Ram Manohar Lohia Combined Hospital, Department Of Anaesthesia, Lucknow, UP, India)
}

\begin{abstract}
Lymphangiomas of the head and neck are sometimes referred to as cystic hygromas and result from abnormal growth of the lymphatic vessels. Lymphangiomas are most commonly found in children and very rarely observed in adults. Most lymphangiomas are congenital, but they have also been reported secondary to infections, neoplasms, trauma, and iatrogenic injuries. The authors report the case of an adult female with a slowly enlarging neck mass that was resected and identified by pathology as a lymphangioma. The literature is reviewed and the classification, symptoms, diagnosis, and treatment of lymphangiomas are discussed. Observation is recommended for small, non-expanding lesions. Surgical resection is recommended for lesions that persist, enlarge, or produce obstructive symptoms. Careful surgical planning is critical to avoid injury to adjacent vital neurovascular structures. A multidisciplinary approach is warranted.
\end{abstract}

Keywords: lymphangioma, cystic hygroma, neck mass

\section{Introduction}

Lymphangiomas are benign lymphatic malformations that occur most commonly in childhood.[1] The initial appearance of cystic hygroma or lymphangioma in adulthood is rare with less than 100 cases reported in literature.[2] Our patient in addition to cervical and axillary involvement had mediastinal involvement on right side. Cervicofacial lymphangioma is noted before birth, at birth, or in the first few months of life in fewer than $50 \%$ of patients. Some authors refer to these lesions as cystic hygromas, a term first used by Wernher in 1843.[3] The term cystic hygroma refers to the cystic nature of these lesions and their mass effect; Hygroma meaning "moist tumor."[2] Lymphangioma, however, is the more appropriate nomenclature because it denotes the tissue of origin.[4] Lymphangiomas are thought to arise from an embryologic defect that consists of an abnormal connection from the jugular sacs to the peripheral lymphatic system.[5]

\section{Llustrative Case}

A 20-year-old female presented to our hospital with a gradually progressive slow-growing neck mass in right supraclavicuar region first noticed one year back. On presentation, the patient noted only slight discomfort and pain in her neck, which occurred with movement. She had no history of shortness of breath, dysphagia, dysphonia.

On physical examination, the mass was approximately $12 \times 12 \mathrm{~cm}$, soft to firm in consistency, immobile, present in inferior part of posterior triangle of neck on right side, with ill-defined margins. There were no signs of inflammation or impulse on cough and the mass was non- pulsatile. There was also ill defined small swelling, $2 \times 2 \mathrm{~cm}$ size present in left supraclavicular region.

Magnetic resonance imaging (MRI) (Figure 1) revealed a large, mass in right side of neck which was extending into supraclavicular region, anterior mediastinum along paratracheal and paraoesophaseal region, right axillary region and encasing axillary vessels neural structures (brachial plexus). The internal carotid artery, internal jugular vein and thyroid were displaced anteriorly while trachea was displaced to the opposite side by the extension into the tracheo-esophaseal groove. Mass was extending into the opposite axillary and supraclavicular region. There was no significant contrast enhancement, and the lesion measured approximately $12 \mathrm{x}$ 12 x $6 \mathrm{~cm}$. Color Doppler revealed a mass with slow flow vascular malformation.

So a pre-operative diagnosis of slow flow arterio-venous malformation was made and a multidisciplinary approach for surgical resection was undertaken with support from ENT, Plastic \& Cardiovascular surgeons. The mass was reached through a reverse $\mathrm{L}$ shaped vertical and transverse skin incision along the posterior border of the sternocleidomastoid muscle. (Figure 2) Although the lesion was in close proximity to nerves, vessels, and muscles, there was no infiltration into these structures, and the mass was circumferentially dissected and total removal was done. Final pathology (Figure 3) revealed a lymphangioma 
with a few small, adjacent benign lymph nodes. The patient tolerated the procedure well and was discharged from the hospital on postoperative day 3 without any neurological deficits.

\section{Discussion}

Lymphangioma is a rare congenital lesion resulting from abnormal growth of the lymphatic vessels. Lymphangiomas are rare, accounting for $4 \%$ of all vascular tumors in children.[8] Although lymphangioma can become evident at any age, 50\% are seen at birth,[7] and $90 \%$ of lymphangiomas are evident by 2 years of age.[7]Initial presentation during adulthood is rare, and fewer than 100 such cases have been reported in English literature. [8]They have no gender predilection or lateralization to either side of the neck.[9]

Although more than $90 \%$ of lymphatic malformations are congenital, but adult presentations may occur secondary to trauma, infection, neoplasms, or iatrogenic injuries.[1] The pathophysiology of adult lymphangiomas is not clearly understood, but these lesions may occur secondary to induction of dormant rests of embryonic lymphatic tissue that are stimulated to differentiate and grow.

Lymphatic malformations can be categorized as superficial cutaneous lymphangioma, cavernous lymphangioma, cystic hygroma, or diffuse systemic lymphangioma.[1,4,10] A subtype, the hemangiolymphangioma is also recognized.[7]Capillary lymphangiomas are composed of small, capillary-sized lymphatic vessels and are characteristically located in the epidermis. Cavernous lymphangiomas are composed of dilated lymphatic channels and characteristically invade surrounding tissues. Cystic hygromas are large, macrocystic lymphangiomas filled with straw-colored, protein-rich fluid. As suggested by their name, hemangiolymphangiomas are lymphangiomas with a vascular component.

Lymphangiomas may also be classified into microcystic, macrocysticand mixed subtypes, according to the size of their cysts.[7]Microcystic lymphangiomas are composed of cysts, each of which measures less than 2 $\mathrm{cm} 3$ in volume. Macrocystic lymphangiomas contain cysts measuring more than $2 \mathrm{~cm} 3$ in volume. Lymphangiomas of the mixed type contain both microcystic and macrocystic components.

Finally, lymphangiomas may be described in stages, which vary by location and extent of disease.[9]

Stage I- Unilateral infrahyoid;

Stage II- Unilateral suprahyoid;

Stage III- Unilateral suprahyoid and infrahyoid;

Stage IV- Bilateral suprahyoid; and

Stage V- Bilateral suprahyoid and infrahyoid.

Most lymphangiomas are asymptomatic. They usually present as a progressively enlarging painless mass. Typically, the mass is soft, non-tender, and ill-defined. Symptoms may develop due to mass effect when the lymphatic malformation enlarges and compresses surrounding vital structures. Obstructive symptoms of dysphagia, dyspnea, or airway compromise may develop. The direct cause of lymphangioma is a blockage of the lymphatic system as the fetus develops. This blockage is thought to be caused by a number of factors, including maternal alcohol use and viral infections during pregnancy. Why the embryonic lymph sacs remain disconnected from the rest of the lymphatic system is not known.[9]Two theories are proposed. The centrifugal theory proposes an abnormal sequestration of primitive lymphatic buds instead of the endothelial channels that proliferate centrifugally from the venous system to become the lymphatic system.[11] The centripetal theory discusses aberrant congenital lymphatic development.[12] The end result for both theories is the inability of the peripheral lymphatics to drain into the jugular sacs. In adults, lymphangiomas may occur from delayed proliferation of cell rests, either spontaneously or in response to infection or trauma.[13] Cystic lymphangioma that emerges during the first two trimesters of pregnancy is associated with genetic disorders such as Noonan syndrome and trisomies 13, 18and 21.Chromosomal aneuploidy such as Turner syndrome or Down syndrome[13] was found in $40 \%$ of patients with cystic hygroma.[15]

Ultrasonography and computed tomography (CT) scanning have been used extensively to evaluate the anatomy of lymphangiomas. On ultrasound examinations, they appear as thin-walled, multiseptate, multicystic, hypoechoic masses.[16] On CT scanning lymphangiomas appear as multiloculated cystic lesions. On T2weighted MRI, lymphangiomas appear isointense to cerebrospinal fluid, whereas their intensity varies on T1weighted images due to variable protein content.[17]Fine-needle aspiration (FNA) is routinely used to evaluate most neck masses. FNA findings include small and round lymphocytes with intermingling histiocytes without mitoses or atypia. In adults with compromised airways, FNA may be therapeutic, as well as diagnostic.[18] Immunohistochemistry may reveal polyclonal B lymphocytes mixed with T cells.[19] Microscopically, these lesions appear as endothelial-lined lymphatic spaces with intervening fibrous tissue and lymphoid aggregates.[20] Various differential diagnoses viz. thymic cyst, pericardial cyst, bronchogenic cyst, cystic teratoma and cystic thymoma, should be kept in mind.[21]

Observation is usually the first step for small, nonexpanding lesions. Those that persist, that continue to grow, or present with obstructive symptoms should be subjected to surgery. Given the proximity of these lesions 
to vital structures of neck and insinuating nature plan for subtotal or near total excision should be tempered with symptomatic recurrence and scarring encountered with reexplorations. Hence, a pragmatic multimodality approach is advocated in treating these lesions. Balakrishnan $\mathrm{K}$ et al consider primary end point of surgery should be functional optimization and symptom reduction rather than aggressive excision.[22] These author argue that patients content with surgery, with lesser or none deficits are better and further justify that residual lesions might regress overtime. However, these regressing lesions are defined with respect to presentation in childhood.[23]To avoid injury to adjacent neurovascular structures, surgical planning is crucial before resection. Some surgeons prefer to use MRI to facilitate resection because it allows improved anatomical demarcation.[1,24] Ultrasonography has limited ability to delineate the anatomical planes of resection or the extent of structural involvement. Complications of resection include infection, bleeding, hematoma, and postoperative seromas. Injury to facial, hypoglossal, glossopharyngeal, recurrent laryngeal, and lingual nerves has been reported.[1,5,25]Complete excision of a lymphangioma has been shown to have an $81 \%$ cure rate. When only part of the lymphatic malformation is excised, there is an $88 \%$ recurrence rate.[6] Several studies have shown an increase in rates of recurrence, morbidity, and complications for lymphangiomas located in the suprahyoid versus infrahyoid region, $[1,25,26]$ whereas one study found no difference in recurrence rates when comparing these locations.[11]However a two-year postsurgical follow-up to detect any recurrence is recommended.[27]Charabi and associates describe a direct correlation between the extent of the lesion and the number of operations performed, rate of recurrence, and residual disease.[26] They state that nonencapsulated lesions recur more frequently because of their tendency to infiltrate major neurovascular structures.[26]

Sclerosing agents and radiation therapy have not been shown to play a role in the primary treatment of cystic hygromas. They may be indicated in lymphatic malformations that are macrocystic and not amenable to surgical resection.[9,20,27,28] Sclerotherapy with OK-432, a lyophilized, low-virulence SU strain of group A Streptococcus pyogenes, has been suggested as a possible therapy for macrocystic lesions. To date, however, the efficacy of OK-432 has not been proven in prospective, controlled trials.[12,28]

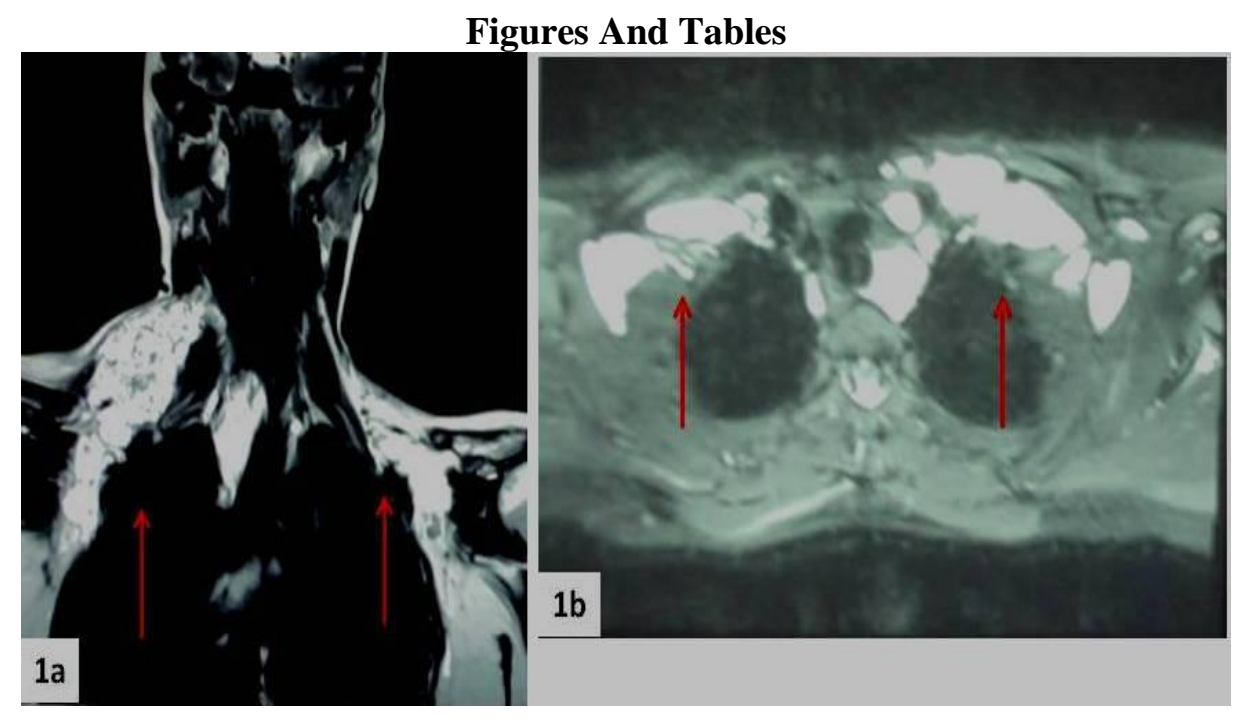

Figure 1: 1a. Coronal 1b. axial MRI of cervico thoracic region show ill defined soft tissue lesion composed of multiple T2 hyperintense cystic spaces situated on right side of neck. Medially lesion extends deep to right common carotid artery and internal jugular vein, right lobe of thyroid gland with extension into tracheoesophageal groove. 


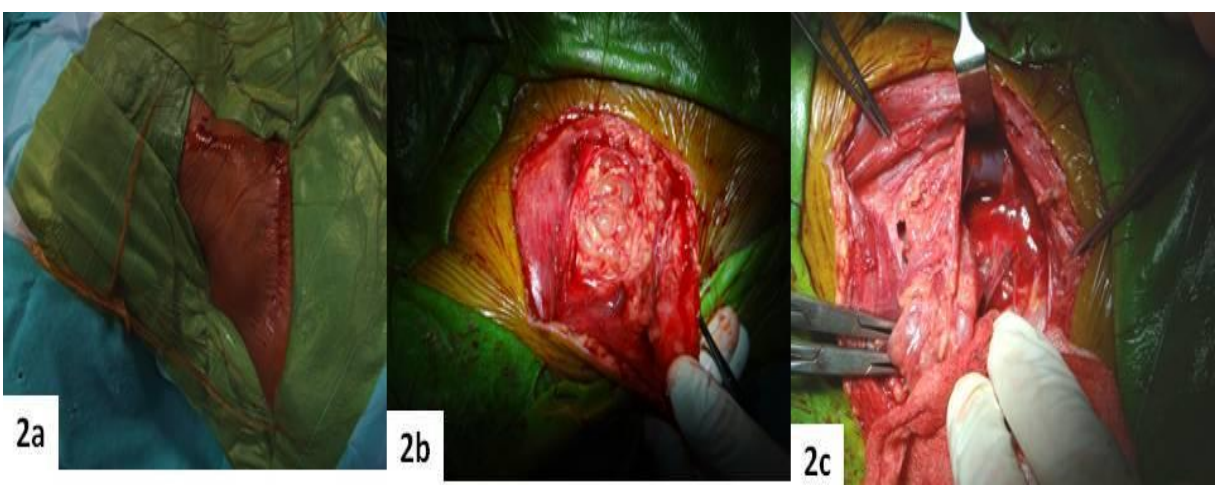

Figure 2: shows operative photograph of excision of lymphangioma. 2a. Incision marked in inverted ' $L$ ' shape. 2b. Exposure of tumor in supraclavicular and cervical region with tumor. Tumor is brownish in color with mulberry like appearance. 2c. Tumor is being excised with well defined plane between tumor and surrounding tissue.
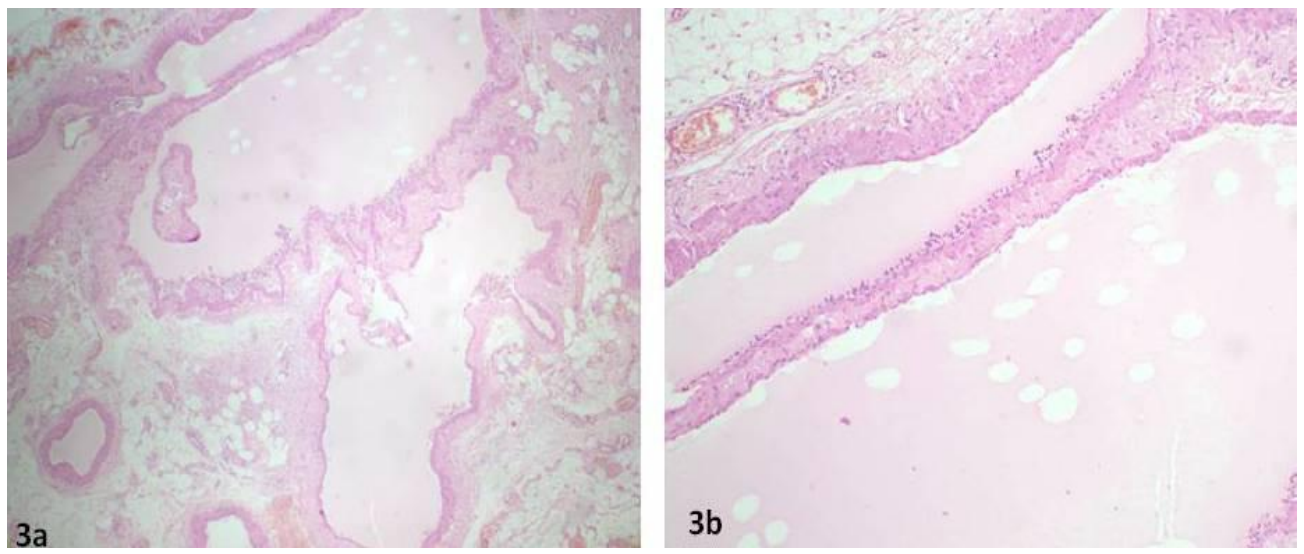

Figure 3: show histopathological appearance of lymphangioma. 3a. multiple dilated lymphatic channels lined by single layer of cuboidal cells and filled with eosinophillic material. Scattered lymphoid cells are also seen within the dialated channels. $3 \mathrm{~b}$. resolved portion of slide depicting lining of cuboidal cells with abundant eosiniphilic material.

nil

\section{Acknowledgements}

\section{References}

[1]. Kennedy TL, Whitaker M, Pellitteri P, et al. Cystic hygroma/ lymphangioma: a rational approach to management. Laryngoscope 2001;111:1929-1937

[2]. Sherman BE, Kendall K. A unique case of the rapid onset of a large cystic hygroma in the adult. Am J Otolaryngol. 2001; 22(3):206-210.

[3]. Wernher A. Die angeborenenZysten-Hygrome und die inhenverwandtenGeschwulste. In: Anatomischer, Diagnostischer und TherapeutischerBeziehung. Geissen: GF Heyer, 1843:76.

[4]. Mulliken JB, Young AE. Lymphatic malformations. In: Vascular Birthmarks: Hemangiomas and Malformations. Philadelphia, Pa: WB Saunders; 1988:41.

[5]. Cheng LH, Wells FC. A multidisciplinary approach to recurrent cervicothoracic cystic hygroma in an adult. Br J Oral Maxillofac Surg 2004;42:66-68.

[6]. Stromberg BV, Weeks PM, Wray RC Jr. Treatment of cystic hygroma. South Med J 1976;69:1333-1335.

[7]. Giguère CM, Bauman NM, Smith RJ. "New treatment options for lymphangioma in infants and children". Ann OtolRhinolLaryngol 2002; 111: 1066-1075.

[8]. Naidu SI, McCalla MR. Lymphatic malformations of the head and neck in adults: A case report and review of the literature. Ann OtolRhinolLaryngol 2004;113:218-222.

[9]. Fernandez, Geover; Robert A Schwartz. eMedicine. Retrieved 2008-11-04.

[10]. Kennedy TL. Cystic hygroma-lymphangioma: a rare and still unclear entity. Laryngoscope 1989;99:1-10.

[11]. Sabin FR. The lymphatic system in human embryos, with a consideration of the morphology of the system as a whole. Am $\mathrm{J}$ Anat 1909;9:43-91

[12]. Sichel JY, Udassin R, Gozal D, et al. OK-432 therapy for cervical lymphangioma. Laryngoscope. 2004;114:1805-09.

[13]. Aneeshkumar MK, Kale S, Kabbani M, et al. Cystic lymphangioma in adults: can trauma be the trigger? Eur Arch Otorhinolaryngol. 2005;262:335-37. 
[14]. Cystic hygroma and lymphangioma: associated findings, perinatal outcome and prognostic factors in live-born infants, Archives of Gynecology and Obstetrics 2007; $276: 491-498$.

[15]. Shulman LP, Emerson DS, Felker RE, Phillips OP, Simpson JL, Elias S. High frequency of cytogenetic abnormalities in fetuses with cystic hygroma diagnosed in the first trimester, Obstetrics Gynecology $199280: 80-82$

[16]. Machado LE, Osborne NG, Bonilla-Musoles F. Three-dimensional sonographic diagnosis of a large cystic neck lymphangioma. J Ultrasound Med 2004;23:877-881.

[17]. Imhof H, Czerny C, Hormann M, et al. Tumors and tumor-like lesions of the neck: from childhood to adult. EurRadiol 2004;14: L155-165.

[18]. Bosch-Princep R, Castellano-Megias VM, Alvaro-Naranjo T, et al. Fine needle aspiration cytology of a cervical lymph node lymphangioma in an adult. A case report. ActaCytol 1999;43: 442-446.

[19]. Fliegelman LJ, Friedland D, Brandwein M, et al. Lymphatic malformation: predictive factors for recurrence. Otolaryngol Head Neck Surg 2000;123:706-710.

[20]. Yildirim E, Dural K, Kaplan T, Sakinci U. Cystic lymphangioma: Report of two atypical cases. InteractCardiovascThorac Surg 2004;3:63-65.

[21]. Fung K, Poenaru D, Soboleski DA, et al. Impact of magnetic resonance imaging on the surgical management of cystic hygromas. J Pediatr Surg 1998;33:839-841.

[22]. Balakrishnan K, Menezes MD, Chen BS, Magit AE, Perkins JA. Primary surgery vs primary sclerotherapy for head and neck lymphatic malformations. JAMA Otolaryngology-Head \& Neck Surgery. 2014;140(1):41-45.

[23]. Perkins JA, Maniglia C, Magit A, et al. Clinical and radiographic findings in children with spontaneous lymphatic malformation regression. Otolaryngol Head Neck Surg. 2008;138(6):772-777.

[24]. Ricciardelli EJ, Richardson MA. Cervicofacial cystic hygroma. Patterns of recurrence and management of the difficult case. Arch Otolaryngol Head Neck Surg 1991;117:546-53.

[25]. Charabi B, Bretlau P, Bille M, et al. Cystic hygroma of the head and neck?a long-term follow-up of 44 cases. Acta Otolaryngol Suppl 2000;543:248-250.

[26]. Chong KT, Ong CL. Cystic hygroma in adulthood. Singapore Med J 1997;38:261-262.

[27]. Mikhail M, Kennedy R, Cramer B, et al. Sclerosing of recurrent lymphangioma using OK-432. J Pediatr Surg 1995;30: 1159-1160.

[28]. Ogita S, Tsuto T, Deguchi E, et al. OK-432 therapy for unresectable lymphangiomas in children. J Pediatr Surg 1991;26: 263-268. 